\title{
Absence of xenotropic murine leukaemia virus- related virus in UK patients with chronic fatigue syndrome
}

\author{
Harriet CT Groom ${ }^{1}$, Virginie C Boucherit ${ }^{1}$, Kerry Makinson², Edward Randal ${ }^{2}$, Sarah Baptista², Suzanne Hagan ${ }^{3}$, \\ John W Gow ${ }^{3}$, Frank M Mattes ${ }^{4}$, Judith Breuer ${ }^{5}$, Jonathan R Kerr², Jonathan P Stoye ${ }^{1}$, Kate N Bishop ${ }^{1 *}$
}

\begin{abstract}
Background: Detection of a retrovirus, xenotropic murine leukaemia virus-related virus (XMRV), has recently been reported in $67 \%$ of patients with chronic fatigue syndrome. We have studied a total of 170 samples from chronic fatigue syndrome patients from two UK cohorts and 395 controls for evidence of XMRV infection by looking either for the presence of viral nucleic acids using quantitative PCR (limit of detection < 16 viral copies) or for the presence of serological responses using a virus neutralisation assay.

Results: We have not identified XMRV DNA in any samples by PCR (0/299). Some serum samples showed XMRV neutralising activity (26/565) but only one of these positive sera came from a CFS patient. Most of the positive sera were also able to neutralise MLV particles pseudotyped with envelope proteins from other viruses, including vesicular stomatitis virus, indicating significant cross-reactivity in serological responses. Four positive samples were specific for XMRV.

Conclusions: No association between XMRV infection and CFS was observed in the samples tested, either by PCR or serological methodologies. The non-specific neutralisation observed in multiple serum samples suggests that it is unlikely that these responses were elicited by XMRV and highlights the danger of over-estimating XMRV frequency based on serological assays. In spite of this, we believe that the detection of neutralising activity that did not inhibit VSV-G pseudotyped MLV in at least four human serum samples indicates that XMRV infection may occur in the general population, although with currently uncertain outcomes.
\end{abstract}

\section{Background}

In 2006, pursuing a link between prostate cancer and an inherited mutation in the RNASEL gene, Urisman and colleagues identified a novel gammaretrovirus [1]. Using PCR methodology, this virus was shown to be present in 9/86 (10\%) prostate tumours examined. It showed close sequence similarity to xenotropic murine endogenous retrovirus elements and was thus named xenotropic murine leukaemia virus related virus (XMRV). A subsequent study demonstrated receptor usage typical of murine xenotropic virus [2]. Phylogenetic analyses place XMRV firmly within the murine endogenous retroviruses [3] even though no identical element has so far

\footnotetext{
* Correspondence: kbishop@nimr.mrc.ac.uk

'Division of Virology, MRC National Institute for Medical Research, The Ridgeway, Mill Hill, London NW7 1AA, UK
}

been identified within the mouse genome [4]. More recently, additional groups of samples from patients with prostate cancer have been examined for the presence of XMRV with both positive [5] and negative [6,7] results.

Very recently, a paper reporting the PCR detection of XMRV in PBMC from 68/101 patients with chronic fatigue syndrome (CFS) has been published [8]. Replicating virus could be isolated from stimulated PBMC with sequences almost, but not quite identical to the viruses isolated from prostate cancer patients. Providing apparently compelling evidence against the possibility of laboratory contamination, a number of the patients were shown to have mounted an immune response against XMRV. Interestingly, around $4 \%$ of control patients appeared to harbour the virus [8]. 
Replication of these results and the possible identification of roles for XMRV in the aetiology of prostate cancer and/or CFS would be of great medical significance. Detection of XMRV might provide potentially useful diagnostic tools and might also suggest therapeutic avenues for treatment. Further, widespread distribution of a potentially pathogenic virus would have important implications concerning its role as a co-factor in other conditions and in the safety of the blood supply. We therefore set out to investigate the distribution of XMRV in UK CFS patients, using PCR to search for the presence of XMRV DNA and neutralisation assays to detect an anti-XMRV immune response. In this study we did not find any association between XMRV infection and CFS.

\section{Methods}

\section{Sample collection}

Samples from the following three centres were tested; St George's University of London (SGUL), Barts and the London Hospital Trust (BLT) and Glasgow Caledonian University (GC).

The SGUL cohort comprised 142 adult CFS patients and 157 healthy blood donors. Both groups were aged between 18 and 65, and the male to female ratios were 45:97 (CFS) and 43:114 (blood donors). At the time of sampling, 2003-2008, blood was collected into three tubes (an EDTA blood tube for DNA preparation; a Paxgene tube for RNA preparation and a plain tube for serum preparation from clotted blood). CFS patients were recruited from clinics in Bristol, Dorset, London, Birmingham, Norfolk and Epsom, and all patients fulfilled diagnostic criteria of Fukuda et al. [9]. Blood samples were taken between 1.5 and 4 years following diagnosis. Healthy normal blood donors were enrolled from the National Blood Service (NBS), in Dorset, UK. All subjects provided informed consent, and these studies were approved by Wandsworth Research Ethics Committee, St George's Hospital, Cranmer Terrace, London SW17 ORE.

The BLT cohort comprised 226 anonymised serum samples taken in 2008-2009 (57 from the antenatal clinic; 58 with haematological disorders; 55 liver patients and 56 from the renal clinic). Clotted blood was separated by centrifugation, and the serum supernatant was removed, stored at $-20^{\circ} \mathrm{C}$ and defrosted once. Ethical approval for the use of these samples for assay development was issued by UCLH NHS trust and adopted by chairman's action at BLT.

The GC cohort comprised 28 CFS patients (20 sera and 8 plasma samples) and 12 controls ( 8 sera and 4 plasma samples) from the West of Scotland catchment area. CFS patients were aged between 28 and 79, with a male to female ratio of $16: 12$. Samples were collected between 1995 and 2003. Controls were aged between 23 and 63 , with a male to female ratio of 7:5. Samples were collected between 2002 and 2004. Some controls were relatives of the patients, and some were hospital staff volunteers. All patients met the Fukuda criteria (9). Ethical permission for blood samples to be analysed for the presence of viruses was granted by Southern General Hospital NHS Trust Local Ethics Committee.

\section{PCR}

Genomic (g)DNA was prepared from PBMC from SGUL patients and controls using the QIAamp DNA mini kit (Qiagen) and amplified using the RepliG Ultrafast Mini Kit (Qiagen), which provides highly uniform amplification of all sequences, with negligible sequence bias. The concentrations after amplification ranged from $108-586 \mathrm{ng} /$ $\mu \mathrm{l}$. Initially, $48 \mathrm{CFS}$ patient gDNA samples were screened by single-round PCR for gag and env genes, as well as GAPDH, as outlined by Lombardi et al. [8] (Table 1). This PCR was performed in a $50 \mu \mathrm{l}$ reaction volume consisting of $25 \mu \mathrm{l}$ amplitaq gold PCR mastermix and a final DNA concentration of 2-5 $\mathrm{ng} / \mu \mathrm{l}$. Cycling was modified as appropriate to our mastermix; $95^{\circ} \mathrm{C}$ for $5 \mathrm{~min},\left(95^{\circ} \mathrm{C}\right.$ for $30 \mathrm{sec}, 57^{\circ} \mathrm{C}$ for $30 \mathrm{sec}$, and $72^{\circ} \mathrm{C}$ for $60 \mathrm{sec}$ ) for $45 \mathrm{cycles}$, hold at $72^{\circ} \mathrm{C}$ for $7 \mathrm{~min}$, store at $4^{\circ} \mathrm{C}$. Products were visualized on $3 \%$ agarose gels by ethidium bromide staining. As we did not amplify any products using this PCR, we developed two more sensitive real-time qPCR assays which targeted 2 regions of the env gene, beginning at nt 6173 and

Table 1 Primer sequences used in XMRV-specific PCRs

\begin{tabular}{|c|c|c|}
\hline Primer & Sequence & Reference \\
\hline 419F gag & ATCAGTTAACCTACCCGAGTCGGAC & $\begin{array}{l}\text { Lombardi } \\
\text { et al, } 2009\end{array}$ \\
\hline 1154R gag & GCCGCCTCTTCTTCATTGTTCT & $\begin{array}{l}\text { Lombardi } \\
\text { et al, } 2009\end{array}$ \\
\hline $5922 \mathrm{~F}$ env & GCTAATGCTACCTCCCTCCTGG & $\begin{array}{l}\text { Lombardi } \\
\text { et al, } 2009\end{array}$ \\
\hline 6273R env & GGAGCCCACTGAGGAATCAAAACAGG & $\begin{array}{l}\text { Lombardi } \\
\text { et al, } 2009\end{array}$ \\
\hline hGAPDH-66F & GAAGGTGAAGGTCGGAGTC & $\begin{array}{l}\text { Lombardi } \\
\text { et al, } 2009\end{array}$ \\
\hline hGAPDH-291R & GAAGATGGTGATGGGATTTC & $\begin{array}{l}\text { Lombardi } \\
\text { et al, } 2009\end{array}$ \\
\hline
\end{tabular}

\begin{tabular}{ll}
\hline Real-time PCR & \\
6173 env $F$ & GGCATACTGGAAGCCATCATCATC \\
6173 env $R$ & CCTGACCCTAAGGAGTTTTCC \\
6173 env probe & ATGGGACCTAATTCC \\
6682 env $F$ & GTGCTGGCTGTGTCTAGTATCG \\
6682 env R & GCAGAGGTATGGTTGAGTAAGTAC \\
6682 env probe & ACGGCCACCCCTTCGT \\
\hline
\end{tabular}


6682, respectively (Table 1 ). These were used to screen samples of gDNA (prepared from PBMC) or cDNA (prepared from total RNA extracted using the Paxgene system from Preanalytix, UK) from CFS and normal blood donors. In total, 136 CFS gDNA and 140 CFS cDNA samples and 95 control gDNA and 141 control cDNA samples were analysed, such that all 142 CFS patients and 157 blood donors were screened for XMRV using these assays in either genomic DNA, cDNA or both. GAPDH was also amplified as a control using a commercial primer and probe set (Hs_99999905_m1 from Applied Biosystems). Real-time qPCR reactions were performed in $10 \mu$ l total volume, consisting of $5 \mu \mathrm{l}$ PCR mastermix, $0.5 \mu \mathrm{l}(20 \times)$ Taqman primers/probe mix, $4.5 \mu \mathrm{l}$ sample (for gDNA, $1 \mu \mathrm{l}$ gDNA (100-590 ng) and 3.5 $\mu$ l DEPC-treated water (Ambion); for cDNA, $4.5 \mu \mathrm{l}$ cDNA). Cycling times and temperatures were as follows. Initial denaturation occurred for $10 \mathrm{~min}$ at $95^{\circ} \mathrm{C}$, followed by 40 cycles of denaturation at $95^{\circ} \mathrm{C}$ for $15 \mathrm{sec}$ and combined primer annealing/extension at $60^{\circ} \mathrm{C}$ for $1 \mathrm{~min}$. Data were displayed using SDS 1.3.1 software (ABI).

\section{Plasmids}

VP62 XMRV clone was a gift of Robert Silverman [2]. HG1 is a replication-incompetent XMRV clone constructed by site-directed mutagenesis of VP62 (the packaging signal was removed by deleting nucleotides 293-388, as numbered in GenBank EF185282; and nucleotides 7720-8108 were replaced by a BsrG1 site to remove the U3 region). Moloney-MLV Gag-Pol was expressed from KB4, a vector synthesized by cloning the gag-pol region from pMD-MLV GagPol [10] into pcDNA3.1. Viral genomic RNA was expressed from an MLV-based retroviral vector encoding $\beta$-galactosidase (LTR-LacZ [10]), and envelope proteins were encoded by constructs for either NZB xenotropic envelope, $\operatorname{MLV}(\mathrm{X})$ (a gift of Massimo Pizzato), Moloney-MLV env (MOSAF, a gift of Yasu Takeuchi), Friend-MLV env [10], or the G-protein from vesicular stomatitis virus (VSV-G) [11].

\section{Virus production}

Replication defective XMRV virus was prepared for neutralisation assays by co-transfecting $293 \mathrm{~T}$ cells with HG1 and LTR-LacZ. Pseudotyped MLV was prepared by co-transfecting $293 \mathrm{~T}$ cells with KB4, LTR-LacZ and an envelope-encoding plasmid (either $\operatorname{MLV}(\mathrm{X}), \mathrm{MOSAF}$, Friend or VSV-G). After $\sim 18$ hours, cells were washed, and fresh media was added for a further $\sim 24$ hours, before viral supernatants were harvested, filtered, and the viral titre was measured by ELISA for RT activity (Cavidi tech). Viral stocks were titrated on D17 cells, an established, easily infectable dog cell line, or NIH-3T3 cells for Friend- and Moloney- pseudotyped MLV. After 48 hours, the cells were assayed for $\beta$-galactosidase activity using the Galacto-Star system (Applied Biosystems). The amount of virus to be used in the neutralisation assays was determined as the volume of supernatant added to $3.5 \times 10^{3}$ cells that resulted in $\sim 4$ $\times 10^{4}$ counts per second of chemiluminescence.

\section{Neutralisation assays}

Neutralisation assays were performed as reported in [12]. Monoclonal antibodies to MLV Env proteins (shown in Table 2) were gifts from Leonard Evans and have been previously described $[13,14]$. They were provided and used as untreated hybridoma cell supernatants that were serially diluted two-fold before adding to virus to assess neutralisation activity as for serum, detailed below. Serum samples were heat inactivated at $56^{\circ} \mathrm{C}$ for $30 \mathrm{~min} .5 \mu \mathrm{l}$ serum were then added to $95 \mu \mathrm{l}$ media in a 96-well tissue culture plate, and samples were serially diluted two-fold, leaving $50 \mu \mathrm{l}$ at each dilution. $50 \mu \mathrm{l}$ virus-containing supernatant were then added to each well, and the plate was incubated at $37^{\circ} \mathrm{C}$ for 1 hour. Following incubation, $100 \mu$ l containing 3.5 $\times 10^{3}$ D17 cells (or NIH-3T3 cells for Friend or Moloney-MLV neutralisation) were added to each well, and the plate was incubated at $37^{\circ} \mathrm{C}$. After 48 hours the cells were lysed, and $\beta$-galactosidase activity was measured. Infectivity corresponded to counts per second of chemiluminescence.

\section{Results}

\section{PCR screening}

Lombardi et al. have recently detected XMRV DNA in $67 \%$ of CFS patients by PCR [8]. To confirm an association of XMRV with this disease, we performed PCR for gag, env and GAPDH on 48 (of 142) CFS patient gDNA samples from SGUL using the previously published

Table 2 Neutralisation properties of different monoclonal antibodies against XMRV and MLV pseudotyped with three different envelopes.

\begin{tabular}{|c|c|c|c|c|c|c|}
\hline \multirow[b]{2}{*}{ Hybridoma $^{1}$} & \multirow[b]{2}{*}{ Raised in } & \multirow[b]{2}{*}{ Isotype } & \multicolumn{4}{|c|}{ Neutralisation of } \\
\hline & & & XMRV & $\operatorname{MLV}(X)$ & Friend & Moloney \\
\hline $83 A 25^{\prime}$ & Rat & $\lg G 2 A$ & Y (88) & Y (89) & ND & $N D$ \\
\hline $24-7$ & Mouse & $\operatorname{lgMK}$ & $N$ & $\mathrm{~N}$ & ND & ND \\
\hline 48 & Mouse & $\lg G 2 A$ & $\mathrm{~N}$ & $\mathrm{~N}$ & Y (95) & Y (83) \\
\hline 538 & Mouse & $\lg M$ & $\mathrm{~N}$ & $\mathrm{~N}$ & $\mathrm{~N}$ & $Y(63)$ \\
\hline 603 & Mouse & $\lg M$ & $\mathrm{~N}$ & Y (96) & $\mathrm{N}$ & ND \\
\hline 609 & Mouse & $\lg M$ & $Y(71)$ & $\mathrm{N}$ & ND & ND \\
\hline 610 & Mouse & $\lg M$ & $N$ & $Y(64)$ & ND & ND \\
\hline 613 & Mouse & $\lg M$ & $\mathrm{~N}$ & Y (91) & ND & ND \\
\hline 615 & Mouse & $\lg M$ & $N$ & $\mathrm{~N}$ & ND & $N D$ \\
\hline
\end{tabular}

$\mathrm{Y}$ indicates neutralisation; $\mathrm{N}$ indicates no neutralisation; ND is not determined The number in brackets refers to the percentage neutralisation at the least diluted antibody concentration.

${ }^{1}$ See references [13] and [14] for description of hybridoma cell lines 
single-round PCR methodology (Table 1 and [8]. However, although all samples were positive for GAPDH, we found no evidence of XMRV DNA in any of the samples (data not shown). In case we were missing low levels of viral DNA, we devised a more sensitive qPCR-based approach. To test the sensitivity of this method, triplicate, serial 1:10 dilutions of VP62 plasmid encoding the full length XMRV genome were added to PBMC DNA from a healthy donor and tested by Taqman PCR with either env 6173 or env 6682 primers (Table 1). All replicates calculated to contain 16 copies of XMRV routinely yielded a product within 37 cycles whereas only one of three replicates of the next dilution scored positive (Figure 1). We concluded that our assay was capable of reliably detecting as little as 16 copies of proviral DNA and was therefore likely to be as sensitive, if not more so, than the assays previously used [8]. We then tested the entire SGUL panel of 142 CFS samples and 157 of the control samples (either gDNA, cDNA or both) with both env 6173 and env 6682 primers. Although positive for GAPDH, all samples were negative for XMRV. To exclude the possibility of specific sample-mediated PCR inhibition, we spiked 3 normal control cDNAs, which had previously tested negative for XMRV nucleic acid, with XMRV VP62 DNA, to a final concentration of 2.3 $\times 10^{-6} \mathrm{ng} / \mu \mathrm{l}$ and repeated the qPCR using both env 6173 and env 6682 primer sets. We successfully amplified the VP62 in these reactions, proving that the PCR should have amplified XMRV from the patient samples if it was present.

\section{Neutralisation assays}

In light of the negative data obtained using PCR assays, we set out to search for evidence of XMRV infection using a second method. Viral infection can elicit a neutralising antibody response [12]. Demonstration of such a neutralising activity can be taken as evidence for a viral infection, perhaps in cell types that were not sampled in blood. Defining neutralisation is difficult in the absence of known positive and negative sera. However, a number of neutralising monoclonal antibodies directed against the Env protein of murine retroviruses have been described $[13,14]$. We therefore obtained several of these (gifts of Leonard Evans) and tested them for neutralisation of XMRV and NZB xenotropic MLV (X) as well as ecotropic Friend and Moloney MLV (Table 2) by assaying for a reduction in virus infectivity following incubation of virus-containing supernatant

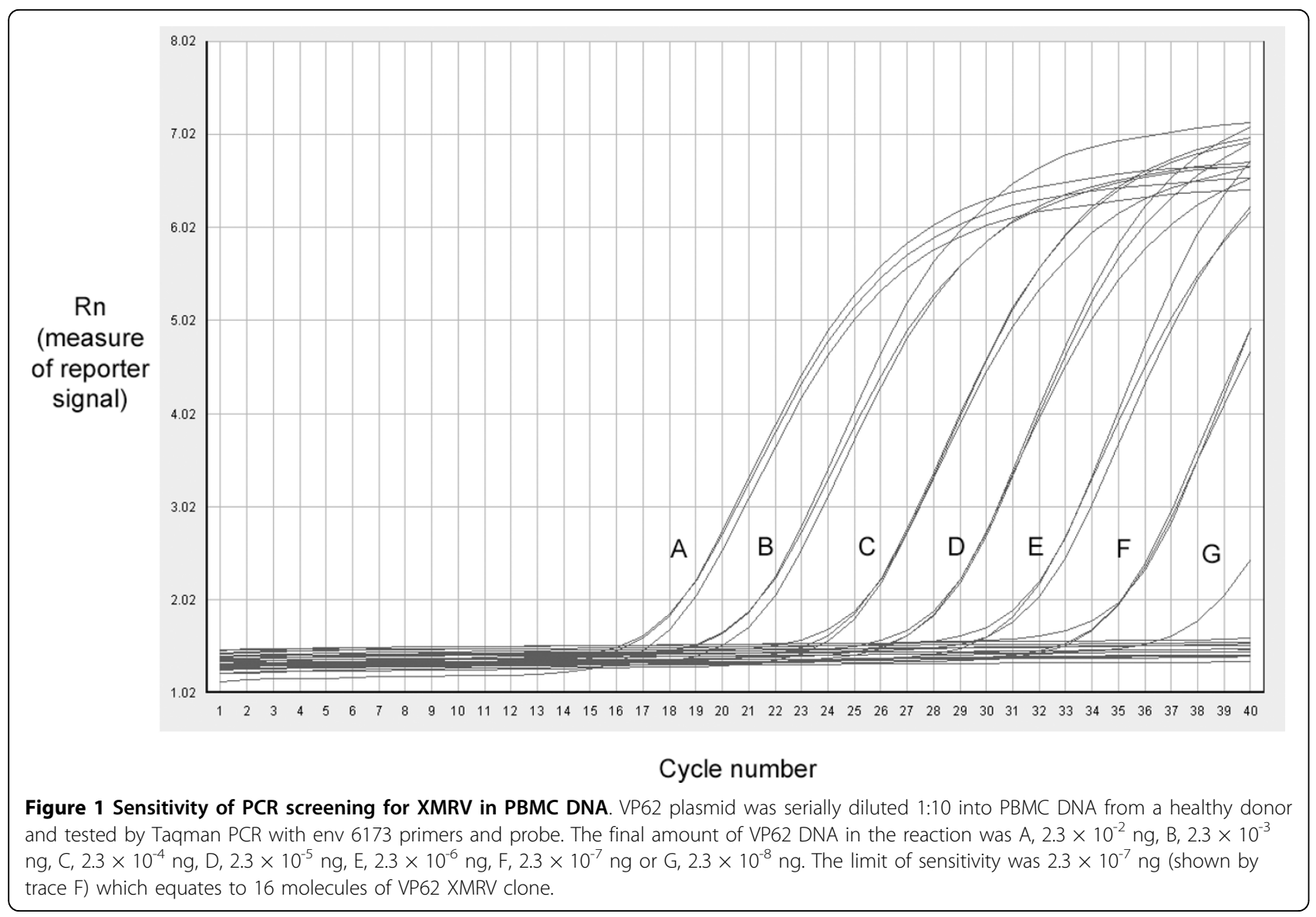


with the monoclonal antibody. As anticipated, some monoclonal antibodies were able to neutralise XMRV (83A25' and 609) whilst others had no effect on XMRV infectivity. Interestingly, we identified three monoclonal antibodies that neutralised $\mathrm{MLV}(\mathrm{X})$ but not XMRV (603, 610 and 613) and one that neutralised XMRV but not $\operatorname{MLV}(X)$ (609). These reagents may therefore be useful tools with which to distinguish XMRV from other xenotropic MLVs in future investigations. From these experiments we defined two negative (603 and 613 ) and one positive (83A25') antibody controls for further experiments. To validate the neutralisation assay and examine the possible range of responses to "normal serum", we tested neutralisation using a panel of 226 serum samples from BLT. Previous investigations have detected XMRV DNA in $~ 1-6 \%$ of control samples $[5,6,8]$. Of our panel only a handful showed possible neutralisation activity, giving curves similar to that shown in Figure 2A, with reductions in viral infectivity similar or greater than that seen with the positive control, monoclonal 83A25'. Over $90 \%$ of the samples tested had less than a 2-fold effect on infectivity (Figure


B
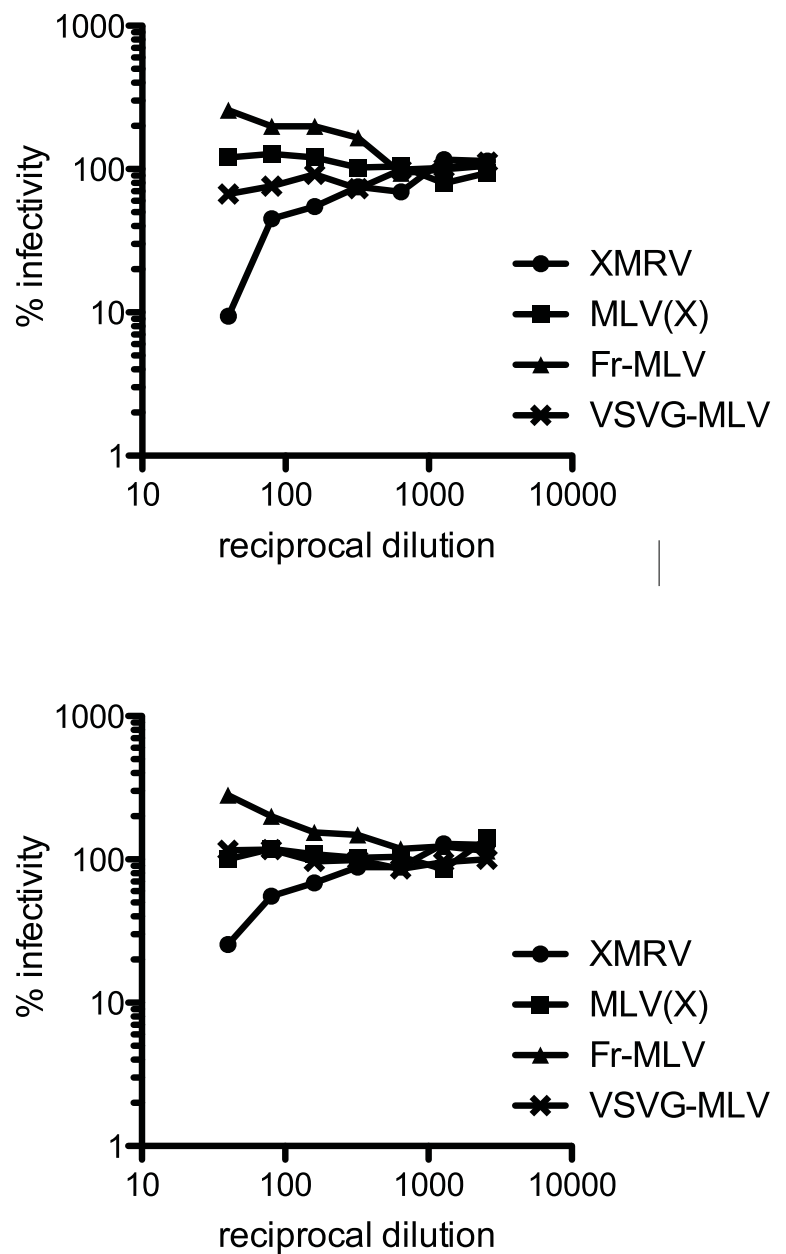

Figure 2 Examples of BLT positive serum neutralisation activity. A, Infectivity of XMRV (measured as counts per second of chemiluminescence produced from $\beta$-galactosidase activity) after incubation with patient serum or hybridoma cell supernatant. Infectivity is plotted against the reciprocal dilution of the BLT serum (black circles, top panel, sample Q488, bottom panel, sample Q610; triangles, negative control, monoclonal 603; squares, positive control, monoclonal 83A25'). The dashed line indicates viral infectivity in the absence of sera. B, Infectivity data for viruses with four different envelopes (circles, XMRV; squares, MLV(X); triangles, Friend-MLV; crosses, VSV-G) after incubation with patient serum. Data were normalised by setting the infectivity for each virus in the absence of patient serum at 100\%, and plotted against the reciprocal of serum dilution for two positive sera, top panel sample Q488 and bottom panel sample Q610. 
3A). From these data, we have defined a positive as a sample that reduces viral infectivity by at least $70 \%$ at a dilution of $1 / 40$ and gives a reduction of $50 \%$ at a $1 / 80$ dilution. According to this definition, the BLT sample set contains 3 neutralising sera, identifying 1.3\% of samples as positive.

To confirm that the neutralisation activity demonstrated was specific for XMRV, we tested a subset of sera for neutralisation of XMRV alongside MLV particles pseudotyped with different envelope proteins from MLV (X), Friend-MLV or VSV. As shown in Figure 2B, of these four virus preparations, only XMRV infectivity was inhibited by any of the sera tested. Even the infectivity of particles expressing the closely related $\operatorname{MLV}(\mathrm{X})$ envelope that is $94 \%$ identical to XMRV was unaffected by sera that inhibited XMRV (Figure 2B, squares). Thus, it seems that the neutralising activity is specific for XMRV.

We therefore felt this assay was sensitive and specific enough to examine the neutralising ability of the SGUL cohort of blinded patient serum samples. After unblinding the samples, it emerged that of the 142 CFS patient sera tested none was positive as defined by the criteria above (Figure 3B). These results suggested that there was no link between XMRV and CFS. By contrast, the control group of 157 blood donors contained 22 positives, a frequency of $14 \%$, considerably higher than that seen in the BLT group (Figure $3 \mathrm{C}$ ). It was also noticeable that the neutralising activity of all but one of the SGUL positive samples was much stronger than the BLT positive samples (compare Figure 2A with Figure 4A). In fact, most of the SGUL positive sera reduced XMRV infectivity by 100 fold at both $1 / 40$ and $1 / 80$ dilutions. Intriguingly, many of these serum samples were collected from a single blood donation session. Some samples from this session, however, were negative. Surprisingly, PCR analyses of DNA samples corresponding to the positive sera from the SGUL controls were uniformly negative. We therefore investigated the specificity of this response by testing 21 of the positive sera for neutralisation of MLV pseudotyped with the envelope proteins from $\mathrm{MLV}(\mathrm{X})$, Friend-MLV or VSV. In every case, the serum was able to neutralise additional viruses to XMRV, including particles pseudotyped with the non-retroviral envelope from VSV (Figure 4B and Table 3). This implied that the strong positive neutralising activity demonstrated by the SGUL blood donor controls was not specific to XMRV, and in all likeliness was not elicited by this virus.

To test whether the SGUL cohort of CFS patients was unique, we also tested 40 samples (including some

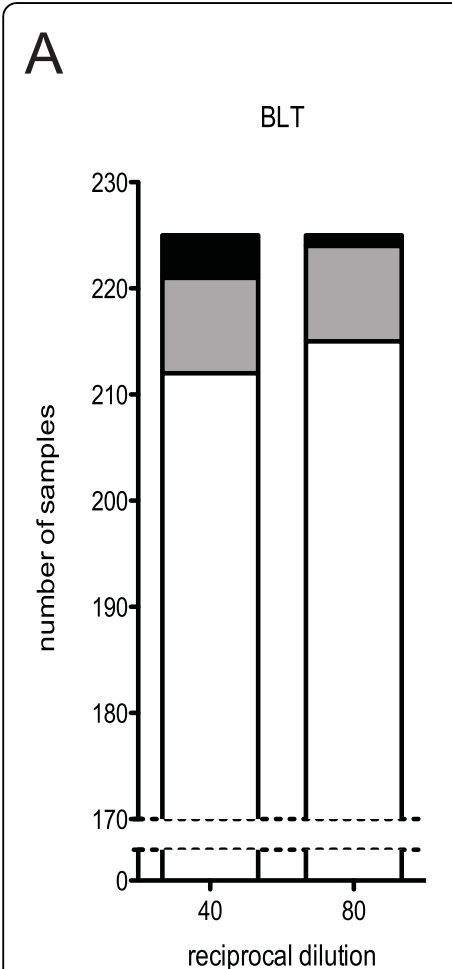

\section{B}



SGUL CFS

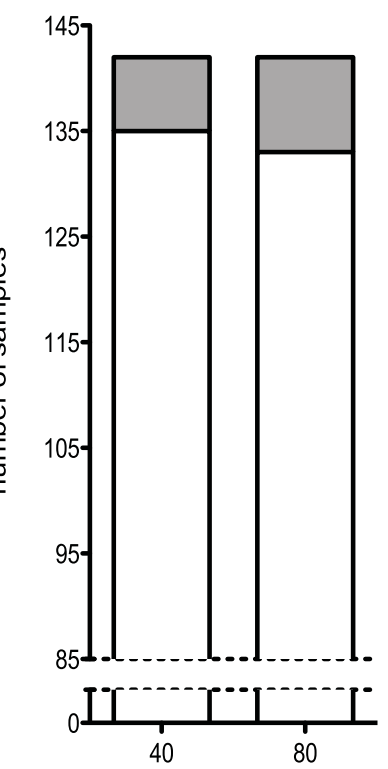

reciprocal dilution
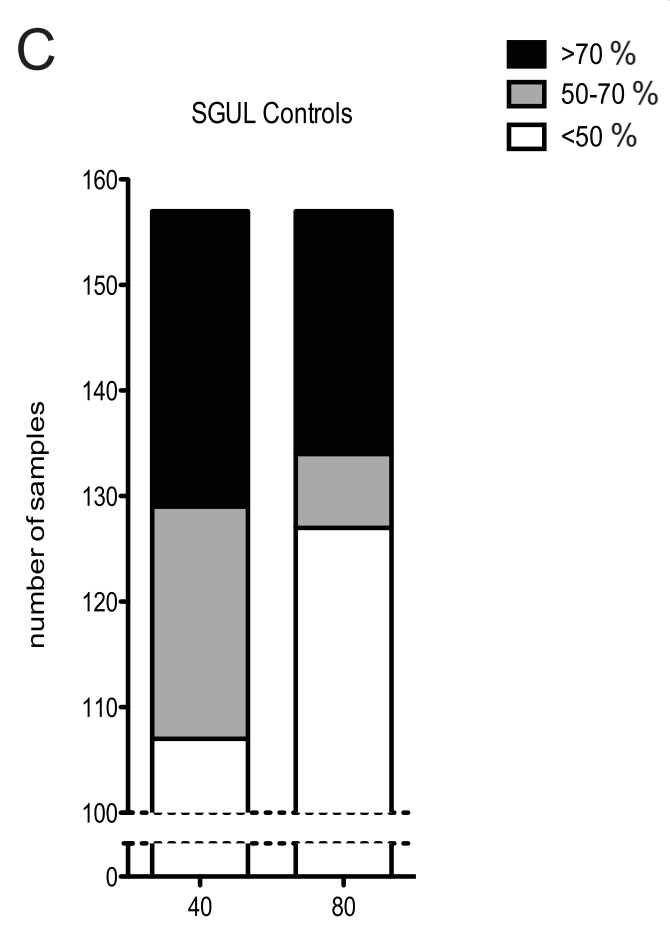

reciprocal dilution

Figure 3 Distribution of neutralisation activity in three samples sets. Numbers of patients showing different degrees (>70\%,50-70\% and $<50 \%)$ of neutralisation of XMRV infectivity are shown for the 1/40 and 1/80 serum dilutions. A, Total BLT cohort $(n=226)$; B, SGUL CFS cohort $(n=142) ; C$, SGUL control blood donor cohort $(n=157)$. 


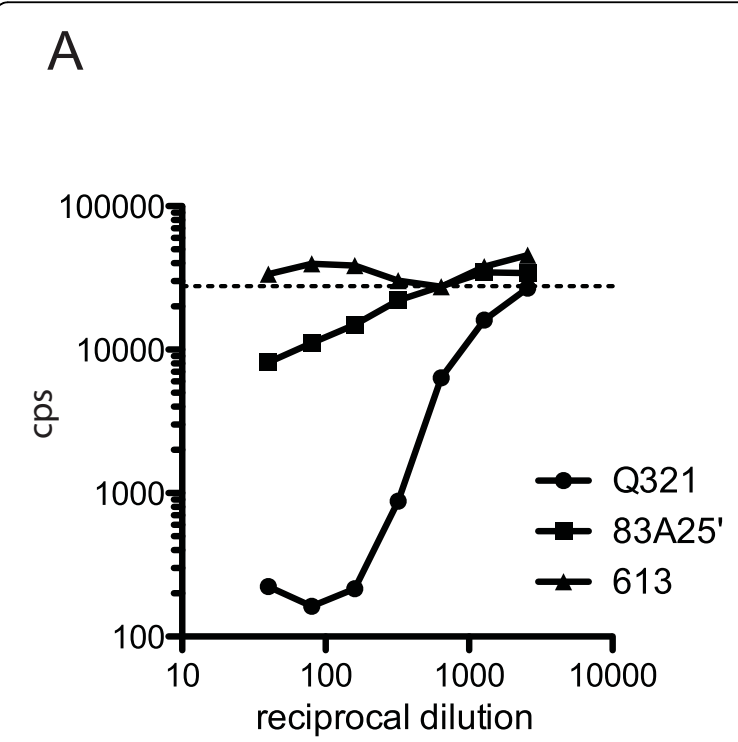

B
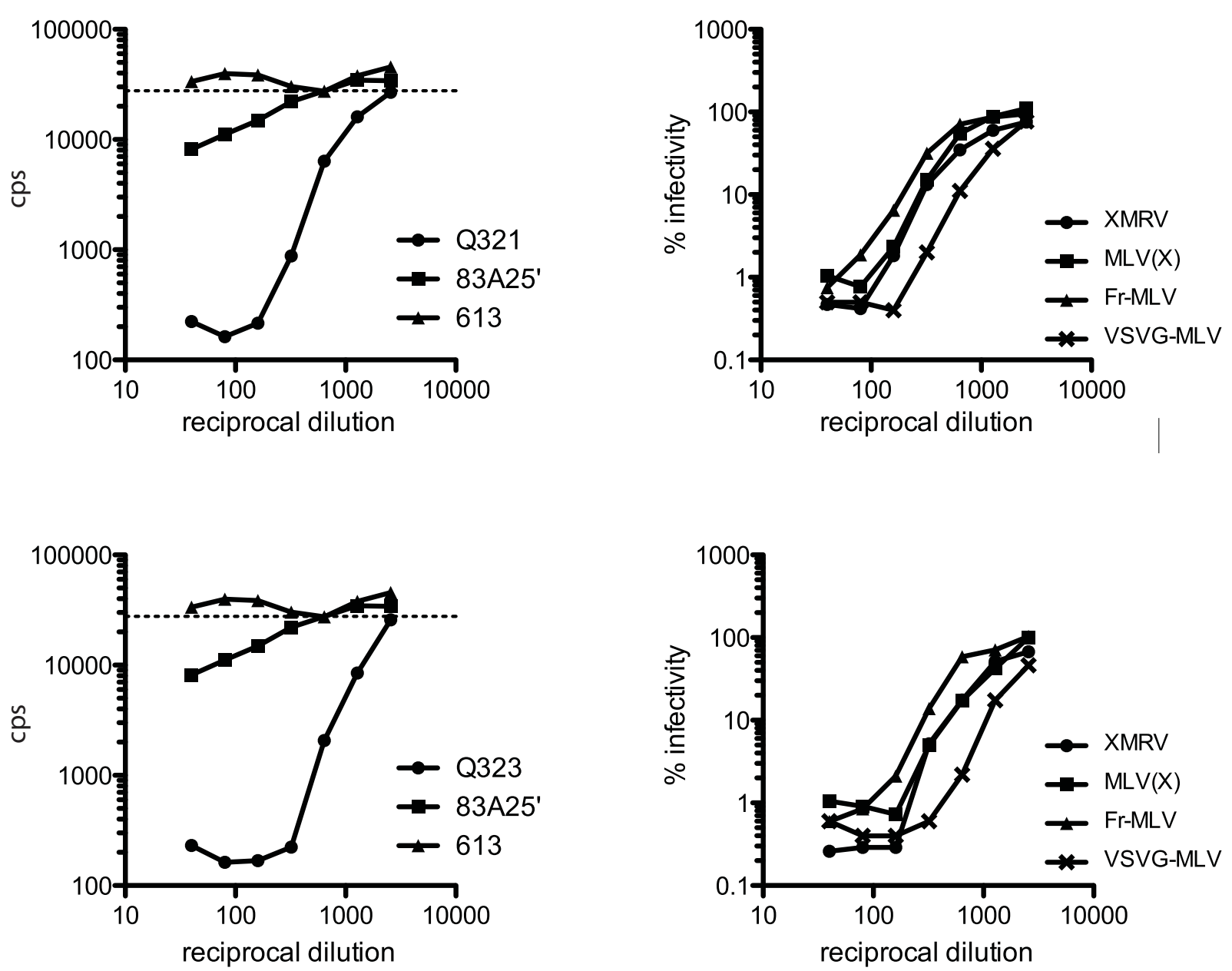

Figure 4 Examples of SGUL positive serum neutralisation activity. A, Infectivity of XMRV (measured as counts per second of chemiluminescence produced from $\beta$-galactosidase activity) after incubation with patient serum or hybridoma cell supernatant. Infectivity is plotted against the reciprocal dilution of the SGUL serum (black circles, top panel, sample Q321, bottom panel, sample Q323; triangles, negative control, monoclonal 613; squares, positive control, monoclonal 83A25'). The dashed line indicates viral infectivity in the absence of sera. B, Infectivity data for viruses with four different envelopes (circles, XMRV; squares, MLV(X); triangles, Friend-MLV; crosses, VSV-G) after incubation with patient serum. Data were normalised by setting the infectivity for each virus in the absence of patient serum at 100\%, and plotted against the reciprocal of serum dilution for two positive sera, top panel sample Q321 and bottom panel sample Q323.

plasma samples as well as sera) from a separate CFS cohort in our neutralisation assay. This GC cohort revealed a solitary positive out of 28 CFS samples (3.6\%), and no positives out of 12 control samples. The positive CFS patient serum was also able to neutralise MLV pseudotyped with either MLV(X) or Friend envelopes, although interestingly, it was not able to neutralise VSV-G pseudotyped MLV (Table 3). Neutralisation data from the different cohorts are summarized in Table 4. Thus, in summary, we found no association of XMRV with either CFS cohort.

\section{Discussion}

We set out with the intention of confirming the results of Lombardi et al. [8] concerning the association of XMRV with CFS. In total, we tested 142 CFS samples for both the presence of XMRV DNA in PBMCs by PCR and for the presence of neutralising antibodies against XMRV in our viral neutralisation assay, and a further 28 CFS samples for neutralising antibodies only. However, in contrast to Lombardi et al., we found no evidence of XMRV DNA in any patient samples tested, and only a single neutralisation-positive patient serum. Our findings 
Table 3 Neutralisation properties of different human sera against XMRV and MLV pseudotyped with three different envelopes.

\begin{tabular}{|c|c|c|c|c|c|}
\hline \multicolumn{6}{|c|}{ Neutralisation of } \\
\hline Sample ID & XMRV & $\operatorname{MLV}(X)$ & Friend & VSV & XMRV detected by PCR \\
\hline \multicolumn{6}{|c|}{ Barts and the London } \\
\hline Q488 & + & - & - & - & ND \\
\hline Q610 & + & - & - & - & ND \\
\hline Q663 & + & ND & ND & ND & ND \\
\hline \multicolumn{6}{|c|}{ St George's University of London } \\
\hline Q302 & ++ & ++ & ++ & ++ & no \\
\hline Q304 & ++ & ++ & ++ & ++ & no \\
\hline Q305 & ++ & ++ & ++ & ++ & no \\
\hline Q306 & ++ & ++ & ++ & ++ & no \\
\hline Q307 & ++ & + & + & - & no \\
\hline Q308 & ++ & ++ & ++ & ++ & no \\
\hline Q309 & ++ & ++ & ++ & ++ & no \\
\hline Q310 & ++ & ++ & ++ & ++ & no \\
\hline Q311 & ++ & + & + & + & no \\
\hline Q312 & ++ & ++ & ++ & ++ & no \\
\hline Q313 & ++ & ++ & ++ & ++ & no \\
\hline Q314 & ++ & ND & ND & ++ & no \\
\hline Q315 & ++ & ++ & ++ & ++ & no \\
\hline Q316 & ++ & ++ & ++ & ++ & no \\
\hline Q317 & ++ & ++ & ++ & ++ & no \\
\hline Q319 & ++ & ND & ND & ++ & no \\
\hline Q320 & ++ & ++ & ++ & ++ & no \\
\hline Q321 & ++ & ++ & ++ & ++ & no \\
\hline Q323 & ++ & ++ & ++ & ++ & no \\
\hline Q324 & ++ & ++ & ++ & ++ & no \\
\hline Q326 & ++ & ND & ND & ND & no \\
\hline Q372 & + & - & - & + & no \\
\hline \multicolumn{6}{|c|}{ Glasgow Calendonian University } \\
\hline Q125 & + & ++ & ++ & - & ND \\
\hline
\end{tabular}

+ indicates neutralising activity; ++ indicates strong neutralising activity; - indicates no neutralising activity; ND is no determined.

therefore appear inconsistent with the previous report that isolated XMRV from PBMCs of CFS patients. We are confident that, although we are unable to replicate the PCR detection of XMRV in PBMC DNA from CFS patients, our PCR assay is more sensitive than the published single round PCR method and should have possessed the necessary sensitivity to detect XMRV if it was indeed present (Figure 1). Furthermore, we were able to detect neutralising activity in one patient and in several control serum samples (Table 4 and Figure 3), implying that our neutralisation assay also has the required sensitivity. The lack of neutralising activity in CFS samples compared to controls could reflect an inability to mount an immune response in these patients. However, in that case, the virus would be expected to replicate to higher levels in CFS patients making it easier to detect by PCR. As we could not detect any evidence of XMRV infection by our PCR assays, we think this is an unlikely explanation. Thus, in our cohorts, we found no association of XMRV with CFS. This is in stark contrast to the result of Lombardi et al. [8]. However, it is thought likely that the term CFS defines multiple diseases [15-17], and it remains formally possible that a fraction of these are associated with XMRV. During the submission of this manuscript another report was published by Erlwein et al. that also failed to detect XMRV in CFS patients by PCR [18]. The publication of these results has promoted much discussion and controversy amongst CFS researchers and patients alike, and has highlighted the need for additional investigations in this area. Following the findings reported here, it would seem a prudent next step for subsequent studies to compare samples and protocols between different laboratories around the world.

There have also been conflicting reports describing the association of XMRV with prostate cancer. Two studies from the USA [1,5] have found an increased 
Table 4 Summary of number of positive sera with XMRV neutralisation properties

\begin{tabular}{lcc}
\hline Sample cohort & Positive & Total number \\
\hline Barts and the London & 3 & 226 \\
$\quad$ Control & & \\
St Georges University of London & 0 & 142 \\
$\quad$ CFS & 22 & 157 \\
Control & & \\
Glasgow Caledonian University & 1 & 28 \\
CFS & 0 & 12 \\
Control & & \\
\hline
\end{tabular}

prevalence of the virus in prostate cancer patients, although they differed as to whether this was dependent on the RNASEL genotype of the patient. Conversely, two German studies failed to establish a link between the virus and disease [6,7]. Nevertheless, XMRV has been detected in the control groups in multiple investigations $[5,6,8]$, with the incidence varying between 1 and $6 \%$. In our serological studies we have also identified neutralising activity against XMRV in around $4 \%$ of all the samples examined. Remarkably many (but not all) of the seropositive samples were identified in a relatively small group of blood donors within the SGUL cohort, possibly suggesting a local outbreak of infection. There is no evidence that this group are related or that they have a particularly high risk of acquiring a retroviral infection. Therefore, an outbreak of this kind seems unlikely. Moreover, all but one of the positive samples from the SGUL set we tested were also able to neutralise MLV pseudotyped with the envelope protein from VSV (Table 3). The one serum that failed to neutralise VSV-G pseudotyped MLV was, however, able to neutralise MLV particles pseudotyped with other retroviral envelopes. We therefore consider these positives from healthy blood donors to be non-specific cross reacting responses. The remaining four positive samples from the BLT and GC cohorts had much weaker neutralisation activities and did not neutralise VSV-G pseudotyped MLV, although, again, the positive serum from GC did neutralise particles expressing other retroviral envelopes (Table 3). Although we cannot rule out the possibility that the activity of these samples against $\mathrm{XMRV}$ is also non-specific, one possible explanation for these serological findings remains that XMRV infection has occurred in around one percent of the population. This figure is consistent with the general prevalence in control samples previously reported. Given the common oncogenic properties of gammaretroviruses [19] and the reported link between XMRV and prostate cancer $[1,5]$, such an observation might be of considerable significance, particularly for the blood transfusion services. It should, however, be noted that we have so far been unable to reliably detect bacterially expressed XMRV Gag proteins by using these sera in immunoblotting experiments. It is therefore conceivable that these neutralising activities were not elicited by XMRV. Further investigations are required to determine the nature of these antiviral activities.

\section{Conclusions}

In summary, we have studied 299 DNA samples and 565 serum samples for evidence of XMRV infection. We have not identified XMRV DNA in any samples by PCR, however, some serum samples were able to neutralise XMRV infectivity in our assay. Only one of these positive sera came from a CFS patient, implying that there is no association between XMRV infection and CFS. Furthermore, most of the positive sera were also able to neutralise MLV particles pseudotyped with other envelope proteins, indicating there may be cross reactivity with other retroviruses and even other enveloped viruses. It therefore seems unlikely that these responses were elicited by XMRV. However, the detection of neutralising activity that did not neutralise VSV-G pseudotyped MLV in at least four human sera may indicate that XMRV infection does occur at in the general population, although the outcome of such infections is currently uncertain.

\section{Acknowledgements}

This work was supported by the UK Medical Research Council (file reference (KB) U117592729 and (JS) U117512710), The Wellcome Trust (grant ID 084955) and CFS Research Foundation, UK. We acknowledge The Cunningham Trust for funding to SH. KNB is a Wellcome Trust Career Development Fellow. We thank Leonard Evans for anti-MLV Env hybridoma supernatants and Robin Weiss and Nigel Temperton for helpful advice about neutralisation assays. We thank the following clinicians who provided patients for the present study; Dr Selwyn Richards, Dr Janice Main, Prof David J Nutt, Dr David Honeybourne, Dr Luis Nacul, Dr Amolak Bansal, Prof Peter Behan and Dr Abhijit Chaudhuri, and Mark Quinlivan for retrieving samples.

\section{Author details}

'Division of Virology, MRC National Institute for Medical Research, The Ridgeway, Mill Hill, London NW7 1AA, UK. ${ }^{2}$ CFS Group, Division of Cellular \& Molecular Medicine, St George's University of London, Cranmer Terrace, London SW17 ORE, UK. ${ }^{3}$ The Centre for Forensic Investigation, Dept of Biological and Biomedical Sciences, Glasgow Caledonian University, Glasgow G4 OBA, UK. ${ }^{4}$ Department of Virology, Barts and The London NHS Trust, 18 Newark St, Whitechapel, London E1 2ES, UK. ${ }^{5}$ Division of Infection and Immunity, University College London, Windeyer Building, 46 Cleveland St London W1T 4JF, UK.

\section{Authors' contributions}

$\mathrm{JK}, \mathrm{JS}$ and $\mathrm{KB}$ conceived and designed the investigation. $\mathrm{HG}$ and VB carried out the viral neutralisation assays and analysed the data. KM, ER, SB and JK performed the PCR analyses. SH, JG, FM, JB and JK provided patient samples. $J S$ and $\mathrm{KB}$ analysed the data and drafted the manuscript. All authors read and approved the final manuscript.

\section{Competing interests}

The authors declare that they have no competing interests. 
Received: 11 January 2010

Accepted: 15 February 2010 Published: 15 February 2010

\section{References}

1. Urisman A, Molinaro RJ, Fischer N, Plummer SJ, Casey G, Klein EA, Malathi K, Magi-Galluzzi C, Tubbs RR, Ganem D, Silverman RH, DeRisi JL: Identification of a novel Gammaretrovirus in prostate tumors of patients homozygous for R462Q RNASEL variant. PLoS Pathog 2006, 2:e25.

2. Dong B, Kim S, Hong S, Das Gupta J, Malathi K, Klein EA, Ganem D, Derisi JL, Chow SA, Silverman RH: An infectious retrovirus susceptible to an IFN antiviral pathway from human prostate tumors. Proc Natl Acad Sci USA 2007, 104:1655-1660.

3. Jern P, Stoye JP, Coffin JM: Role of APOBEC3 in genetic diversity among endogenous murine leukemia viruses. PLoS Genet 2007, 3:2014-2022

4. Coffin JM, Stoye JP: Virology. A new virus for old diseases?. Science 2009, 326:530-531.

5. Schlaberg R, Choe DJ, Brown KR, Thaker HM, Singh IR: XMRV is present in malignant prostatic epithelium and is associated with prostate cancer, especially high-grade tumors. Proc Natl Acad Sci USA 2009, 106:16351-16356.

6. Fischer N, Hellwinkel O, Schulz C, Chun FK, Huland H, Aepfelbacher M, Schlomm T: Prevalence of human gammaretrovirus XMRV in sporadic prostate cancer. J Clin Virol 2008, 43:277-283.

7. Hohn O, Krause H, Barbarotto P, Niederstadt L, Beimforde N, Denner J, Miller K, Kurth R, Bannert N: Lack of evidence for xenotropic murine leukemia virus-related virus (XMRV) in German prostate cancer patients. Retrovirology 2009, 6:92.

8. Lombardi VC, Ruscetti FW, Das Gupta J, Pfost MA, Hagen KS, Peterson DL, Ruscetti SK, Bagni RK, Petrow-Sadowski C, Gold B, Dean M, Silverman RH, Mikovits JA: Detection of an Infectious Retrovirus, XMRV, in Blood Cells of Patients with Chronic Fatigue Syndrome. Science 2009, 326:585-589.

9. Fukuda K, Straus SE, Hickie I, Sharpe MC, Dobbins JG, Komaroff A: The chronic fatigue syndrome: a comprehensive approach to its definition and study. International Chronic Fatigue Syndrome Study Group. Ann Intern Med 1994, 121:953-959.

10. Mothes W, Boerger AL, Narayan S, Cunningham JM, Young JA: Retroviral entry mediated by receptor priming and low $\mathrm{pH}$ triggering of an envelope glycoprotein. Cell 2000, 103:679-689.

11. Bock M, Bishop KN, Towers G, Stoye JP: Use of a transient assay for studying the genetic determinants of Fv1 restriction. J Virol 2000, 74:7422-7430.

12. Temperton NJ, Hoschler K, Major D, Nicolson C, Manvell R, Hien VM, Ha do $Q$, de Jong $M$, Zambon $M$, Takeuchi $Y$, Weiss RA: A sensitive retroviral pseudotype assay for influenza H5N1-neutralizing antibodies. Influenza Other Respi Viruses 2007, 1:105-112.

13. Chesebro B, Britt W, Evans L, Wehrly K, Nishio J, Cloyd M: Characterization of monoclonal antibodies reactive with murine leukemia viruses: use in analysis of strains of Friend MCF and Friend ecotropic murine leukemia virus. Virology 1983, 127:134-148.

14. Evans LH, Morrison RP, Malik FG, Portis J, Britt WJ: A neutralizable epitope common to the envelope glycoproteins of ecotropic, polytropic, xenotropic, and amphotropic murine leukemia viruses. J Virol 1990, 64:6176-6183.

15. Hickie I, Davenport T, Vernon SD, Nisenbaum R, Reeves WC, HadziPavlovic D, Lloyd A: Are chronic fatigue and chronic fatigue syndrome valid clinical entities across countries and health-care settings?. Aust N Z J Psychiatry 2009, 43:25-35.

16. Kato K, Sullivan PF, Evengard B, Pedersen NL: A population-based twin study of functional somatic syndromes. Psychol Med 2009, 39:497-505.

17. Kerr JR, Petty R, Burke B, Gough J, Fear D, Sinclair LI, Mattey DL, Richards SC, Montgomery J, Baldwin DA, Kellam P, Harrison TJ, Griffin GE, Main J, Enlander D, Nutt DJ, Holgate ST: Gene expression subtypes in patients with chronic fatigue syndrome/myalgic encephalomyelitis. J Infect Dis 2008, 197:1171-1184

18. Erlwein O, Kaye S, McClure MO, Weber J, Wills G, Collier D, Wessely S, Cleare A: Failure to Detect the Novel Retrovirus XMRV in Chronic Fatigue Syndrome. PLOS ONE 2010, 5:e8519.

19. Rosenberg N, Jolicoeur P: Retroviral pathogenesis. Retroviruses Cold Spring Harbor Press, Cold Spring Harbor, NYCoffin JM, Hughes SH, Varmus HE 1997, Chapter 10:475-585. doi:10.1186/1742-4690-7-10

Cite this article as: Groom et al:: Absence of xenotropic murine leukaemia virus-related virus in UK patients with chronic fatigue syndrome. Retrovirology 2010 7:10.

\section{Submit your next manuscript to BioMed Central and take full advantage of:}

- Convenient online submission

- Thorough peer review

- No space constraints or color figure charges

- Immediate publication on acceptance

- Inclusion in PubMed, CAS, Scopus and Google Scholar

- Research which is freely available for redistribution

Submit your manuscript at www.biomedcentral.com/submit
C Biomed Central 